\title{
An efficient one-pot multicomponent synthesis and characterization of 5-cyano-1,2,3,4-tetrahydropyrimidine derivatives
}

\author{
R. G. Vaghasiya, V. H. Shah* \\ Department of Chemistry, Saurashtra University, University Road, Rajkot - 360 005, Gujarat, India \\ *E-mail address: rgvaghasiya409@gmail.com , shah_v_h@yahoo.com
}

\begin{abstract}
A series of pyrimidine-5-carbonitrile derivatives was efficiently synthesized via one-pot, multi component reaction (MCRs) of 1,3-bifunctional synthon (ethylcyano acetate), substituted pyrazole-4carbaldehyde, and thiourea/urea in the presence of basic catalyst. The key advantages of this process are high yields, shorter reaction times, easy work-up, and purification of products by nonchromatographic method.
\end{abstract}

Keywords: substituted pyrazole-4-carbaldehyde; 5-cyano-1,2,3,4-tetrahydropyrimidine derivatives; multi component reaction; non-chromatographic method

\section{INTRODUCTION}

Multi component reactions (MCRs) have more importance, great interest enjoying an outstanding status in modern organic synthesis and medicinal chemistry because they are onepot processes bringing together three or more components and show high atom economy and high selectivity. ${ }^{1,2}$ MCRs comply with the principles of green chemistry in terms of economy of steps as well as many of the rigid criteria of an ideal organic synthesis. These reactions are effective in construction highly functionalized small organic molecules from readily available starting materials in a single step with natural flexibility for creating molecular complexity and diversity coupled with minimization of time, labor, cost and waste production. ${ }^{3}$ The literature survey indicated that a wide range of pharmacological activities are exhibited by the compounds encompassing pyrimidines nucleus. In addition to this, various analogs of pyrimidines have been found to posses antibacterial, ${ }^{4}$ antifungal, ${ }^{5}$ antileishmanial, ${ }^{6}$ antiinflammatory, ${ }^{7}$ analgesic, ${ }^{8}$ antihypertensive, ${ }^{9}$ antipyretic, ${ }^{10}$ antiviral, ${ }^{11}$ antidiabetic, ${ }^{12}$ antiallerggic, ${ }^{13}$ anticonvulsant, ${ }^{14}$ antioxidant, ${ }^{15}$ antihistaminic, ${ }^{16}$ herbicidal, ${ }^{17}$ and anticancer activities $^{18}$ and many of pyrimidines derivatives are reported to possess potential central nervous system (CNS) depressant properties ${ }^{19}$ and also act as calcium channel blockers. ${ }^{20}$

In a view of great importance of pyrimidines derivatives, various methods for synthesis of pyrimidines derivatives have been reported. During the last few years, some methods were introduced for the synthesis of these compounds. Many methods for synthesis of 5-cyano1,2,3,4-tetrahydropyrimidine derivatives have been reported using various catalyst such as 
Magnesium methoxide $\left(\mathrm{Mg}(\mathrm{OMe})_{2}\right),{ }^{21}$ piperidine, ${ }^{22}$ sodium ethoxide, ${ }^{23}$ and also under microwave irradiation. ${ }^{24}$

\section{RESULT AND DISCUSSION}

\section{1. Chemistry}

The synthesis 5-cyano-1,2,3,4-tetrahydropyrimidine derivatives was performed as shown in Scheme 1. In the initial step, substituted pyrazole-4-carbaldehyde was synthesized by Vilsmeier-Haack formylation of hydrazone derivatives of phenyl hydrazine and substituted acetophenones. The 5-cyano-1,2,3,4-tetrahydropyrimidine derivatives (RGV-231 to 250) were synthesized by the reaction of the substituted pyrazole-4-carbaldehyde, ethylcyano acetate and thiourea/urea in the presence of $\mathrm{K}_{2} \mathrm{CO}_{3}$ in ethanol at reflux temperature. The purity of the compounds was determined by TLC and elemental analyses.

\section{Scheme 1}<smiles>[R]c1nn(-c2ccccc2)cc1C=O</smiles>

$\mathrm{R}=$ various substituted phenyl group<smiles>[R]c1nn(-c2ccccc2)cc1C1=C(C#N)C(=O)NC(=[X])N1</smiles>

\section{RGV-231 to 250}

Reagents and conditions: (a) EtOH, $\mathrm{K}_{2} \mathrm{CO}_{3}$, reflux, $4 \mathrm{~h}$.

Table 1. Synthesis of 5-cyano-1,2,3,4-tetrahydropyrimidine derivatives.

\begin{tabular}{|c|c|c|c|c|c|c|}
\hline Code & $\mathbf{R}_{\mathbf{1}}$ & $\mathbf{X}$ & M.F. & M.W. & M.P. ${ }^{\circ} \mathbf{C}$ & $\mathbf{R}_{\mathbf{f}}$ \\
\hline $\mathrm{RGV}-231$ & $4-\mathrm{NO}_{2}-\mathrm{C}_{6} \mathrm{H}_{5}$ & $\mathrm{~S}$ & $\mathrm{C}_{20} \mathrm{H}_{12} \mathrm{~N}_{6} \mathrm{O}_{3} \mathrm{~S}$ & 416 & $170-172$ & 0.55 \\
\hline $\mathrm{R} G V-232$ & $3-\mathrm{NO}_{2}-\mathrm{C}_{6} \mathrm{H}_{5}$ & $\mathrm{~S}$ & $\mathrm{C}_{20} \mathrm{H}_{12} \mathrm{~N}_{6} \mathrm{O}_{3} \mathrm{~S}$ & 416 & $184-186$ & 0.51 \\
\hline $\mathrm{R} G V-233$ & $4-\mathrm{Cl}_{-} \mathrm{C}_{6} \mathrm{H}_{5}$ & $\mathrm{~S}$ & $\mathrm{C}_{20} \mathrm{H}_{12} \mathrm{ClN}_{5} \mathrm{OS}$ & 405 & $168-170$ & 0.61 \\
\hline $\mathrm{RGV}-234$ & $4-\mathrm{OCH}_{3}-\mathrm{C}_{6} \mathrm{H}_{5}$ & $\mathrm{~S}$ & $\mathrm{C}_{21} \mathrm{H}_{15} \mathrm{~N}_{5} \mathrm{O}_{2} \mathrm{~S}$ & 401 & $154-156$ & 0.57 \\
\hline $\mathrm{RGV}-235$ & $4-\mathrm{CH}_{3}-\mathrm{C}_{6} \mathrm{H}_{5}$ & $\mathrm{~S}$ & $\mathrm{C}_{21} \mathrm{H}_{15} \mathrm{~N}_{5} \mathrm{OS}$ & 385 & $174-176$ & 0.48 \\
\hline $\mathrm{RGV}-236$ & $4-\mathrm{F}-\mathrm{C}_{6} \mathrm{H}_{5}$ & $\mathrm{~S}$ & $\mathrm{C}_{20} \mathrm{H}_{12} \mathrm{FN} \mathrm{N}_{5} \mathrm{OS}$ & 389 & $182-184$ & 0.60 \\
\hline
\end{tabular}




\begin{tabular}{|c|c|c|c|c|c|c|}
\hline RGV-237 & $4-\mathrm{Br}-\mathrm{C}_{6} \mathrm{H}_{5}$ & $\mathrm{~S}$ & $\mathrm{C}_{20} \mathrm{H}_{12} \mathrm{BrN}_{5} \mathrm{OS}$ & 450 & $188-190$ & 0.52 \\
\hline RGV-238 & $\mathrm{C}_{6} \mathrm{H}_{5}$ & $\mathrm{~S}$ & $\mathrm{C}_{20} \mathrm{H}_{13} \mathrm{~N}_{5} \mathrm{OS}$ & 371 & $156-158$ & 0.62 \\
\hline RGV-239 & $2-\mathrm{OCH}_{3}-\mathrm{C}_{6} \mathrm{H}_{5}$ & $\mathrm{~S}$ & $\mathrm{C}_{21} \mathrm{H}_{15} \mathrm{~N}_{5} \mathrm{O}_{2} \mathrm{~S}$ & 401 & $148-150$ & 0.50 \\
\hline RGV-240 & $2,4-\mathrm{di}-\mathrm{Cl}-\mathrm{C}_{6} \mathrm{H}_{4}$ & $\mathrm{~S}$ & $\begin{array}{c}\mathrm{C}_{20} \mathrm{H}_{11} \mathrm{Cl}_{2} \mathrm{~N}_{5} \mathrm{O} \\
\mathrm{S}\end{array}$ & 440 & $198-200$ & 0.56 \\
\hline RGV-241 & $4-\mathrm{NO}_{2}-\mathrm{C}_{6} \mathrm{H}_{5}$ & $\mathrm{O}$ & $\mathrm{C}_{20} \mathrm{H}_{12} \mathrm{~N}_{6} \mathrm{O}_{4}$ & 400 & $174-176$ & 0.54 \\
\hline RGV-242 & $3-\mathrm{NO}_{2}-\mathrm{C}_{6} \mathrm{H}_{5}$ & $\mathrm{O}$ & $\mathrm{C}_{20} \mathrm{H}_{12} \mathrm{~N}_{6} \mathrm{O}_{4}$ & 400 & $180-182$ & 0.55 \\
\hline RGV-243 & $4-\mathrm{Cl}-\mathrm{C}_{6} \mathrm{H}_{5}$ & $\mathrm{O}$ & $\mathrm{C}_{20} \mathrm{H}_{12} \mathrm{ClN}_{5} \mathrm{O}_{2}$ & 389 & $164-166$ & 0.50 \\
\hline RGV-244 & $4-\mathrm{OCH}_{3}-\mathrm{C}_{6} \mathrm{H}_{5}$ & $\mathrm{O}$ & $\mathrm{C}_{21} \mathrm{H}_{15} \mathrm{~N}_{5} \mathrm{O}_{3}$ & 385 & $150-152$ & 0.61 \\
\hline RGV-245 & $4-\mathrm{CH}_{3}-\mathrm{C}_{6} \mathrm{H}_{5}$ & $\mathrm{O}$ & $\mathrm{C}_{21} \mathrm{H}_{15} \mathrm{~N}_{5} \mathrm{O}_{2}$ & 369 & $170-172$ & 0.65 \\
\hline RGV-246 & $4-\mathrm{F}-\mathrm{C}_{6} \mathrm{H}_{5}$ & $\mathrm{O}$ & $\mathrm{C}_{20} \mathrm{H}_{12} \mathrm{FN}_{5} \mathrm{O}_{2}$ & 373 & $156-158$ & 0.44 \\
\hline RGV-247 & $4-\mathrm{Br}-\mathrm{C}_{6} \mathrm{H}_{5}$ & $\mathrm{O}$ & $\mathrm{C}_{20} \mathrm{H}_{12} \mathrm{BrN}_{5} \mathrm{O}_{2}$ & 434 & $178-180$ & 0.56 \\
\hline RGV-248 & $\mathrm{C}_{6} \mathrm{H}_{5}$ & $\mathrm{O}$ & $\mathrm{C}_{20} \mathrm{H}_{13} \mathrm{~N}_{5} \mathrm{O}_{2}$ & 355 & $160-162$ & 0.60 \\
\hline RGV-249 & $2-\mathrm{OCH}_{3}-\mathrm{C}_{6} \mathrm{H}_{5}$ & $\mathrm{O}$ & $\mathrm{C}_{21} \mathrm{H}_{15} \mathrm{~N}_{5} \mathrm{O}_{3}$ & 385 & $142-144$ & 0.58 \\
\hline RGV-250 & $2,4-\mathrm{di}-\mathrm{Cl}-\mathrm{C}_{6} \mathrm{H}_{4}$ & $\mathrm{O}$ & $\mathrm{C}_{20} \mathrm{H}_{11} \mathrm{Cl}_{2} \mathrm{~N}_{5} \mathrm{O}_{2}$ & 424 & $190-192$ & 0.54 \\
\hline
\end{tabular}

TLC Solvent system: Hexane: Ethyl acetate - 4:6.

Table 2. Effects of different catalysts on the reaction productivity.

\begin{tabular}{|c|c|c|c|}
\hline Entry & Catalyst & Time (h) & Yield $^{\mathbf{b}}(\mathbf{\%})$ \\
\hline 1 & Piperidine & 6 & 78 \\
\hline 2 & $\mathrm{C}_{2} \mathrm{H}_{5} \mathrm{ONa}$ & 10 & 70 \\
\hline 3 & $\mathrm{~K}_{2} \mathrm{CO}_{3}{ }^{\mathrm{a}}$ & 4 & 90 \\
\hline 4 & $\mathrm{NaOH}$ & 8 & 82 \\
\hline 5 & $\left(\mathrm{C}_{2} \mathrm{H}_{5}\right)_{2} \mathrm{NH}$ & 12 & 80 \\
\hline
\end{tabular}

${ }^{a}$ Reaction conditions: Ethylcyanoacetate $(1.2 \mathrm{mmol})$,

3-(4-chlorophenyl)-1-phenyl-1H-pyrazole-4-carbaldehyde (1.0 mmol), thiourea $(1.5 \mathrm{mmol})$ in ethanol at reflux temperature.

${ }^{\mathrm{b}}$ Isolated Yields.

Several methods are used in the synthesis of these 5-cyano-1,2,3,4tetrahydropyrimidines. The synthesis of these heterocycles has been usually carried out in polar protic organic solvents such as water, methanol and polar aprotic organic solvents such as acetonitrile, DMF and DMSO. The choice of a solvent is a crucial factor for 
multicomponent reactions. So at the first step we looked into the solvent selection for this reaction. We had selected protic solvent ethanol for this reaction. A part from the solvent, the efficiency of the multicomponent reactions is mainly affected by the catalyst and the reaction time. To evaluate the catalytic effect of various catalysts we started with the model reaction of ethylcyanoacetate $(0.03$ mole) with 3-(4-chlorophenyl)-1-phenyl-1H-pyrazole-4-carbaldehyde (0.03 mole) and thiourea ( 0.03 mole) in ethanol with use of various catalysts to afford pyrimidine derivatives in various yields (Table 2). It can be seen from Table 2 that potassium carbonate $\left(\mathrm{K}_{2} \mathrm{CO}_{3}\right)$ is the most efficient (Table 2, entry 3 ) among the all catalysts studied.

In order to evaluate the generality of this model reaction we then prepared a range of 5 cyano-1,2,3,4-tetrahydropyrimidine derivatives under the optimized reaction conditions. In all cases, aryl aldehydes with substituents carrying either electron-donating or electronwithdrawing groups reacted successfully and gave the expected products in good to excellent yields in relatively short reaction times. The kind of aldehyde has no significant effect on the reaction. The results are shown in Table 1.<smiles>[Y]C(N)=[V]CCC[Al]=C(C#N)C(=O)OCC</smiles>

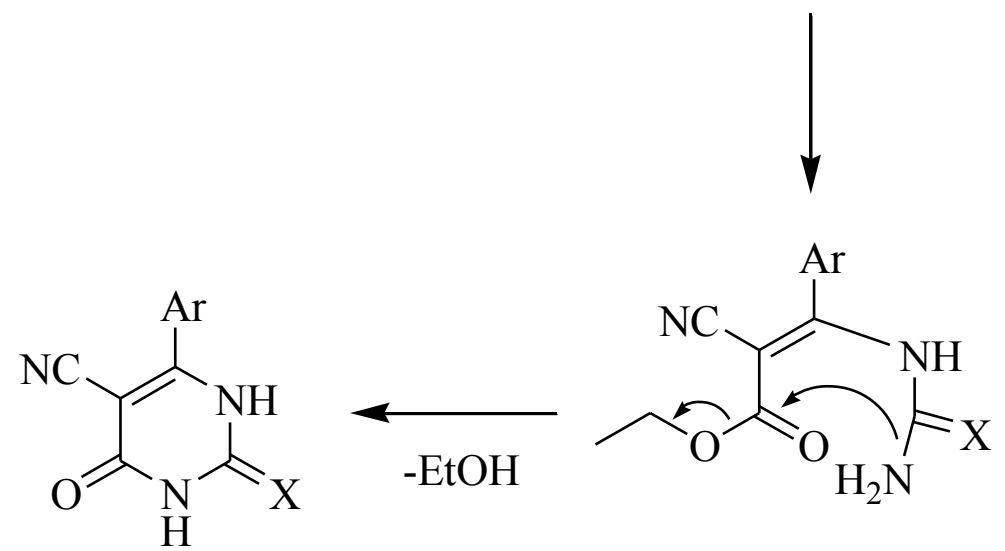

Scheme 2. Plausible mechanism for the formation of substituted pyrano[2,3-b]indoles using base as catalyst.

Possible mechanism for the base catalyzed synthesis of substituted pyrano[2,3-b]indoles has been proposed in Scheme 2. In summary, this paper describes a convenient and efficient process for the synthesis of 5-cyano-1,2,3,4-tetrahydropyrimidine derivatives through the three components coupling of substituted pyrazole-4-carbaldehyde, ethylcyano acetate and thiourea/urea in the presence of $\mathrm{K}_{2} \mathrm{CO}_{3}$ in ethanol at reflux temperature. Reaction profile is very clean and no side products are formed. All the synthesized 5-cyano-1,2,3,4tetrahydropyrimidines have been characterized on the basis of elemental and spectral studies. 


\section{EXPERIMENTAL SECTION}

All research chemicals were purchased from Sigma-Aldrich and used as such for the reactions. Reactions were monitored by thin-layer chromatography (TLC) on pre-coated silica gel GF254 plates from E-Merck Co and compounds visualized either by exposure to UV light or staining with reagents. Melting points were determined in open capillaries and are uncorrected. The IR spectra were recorded on SHIMADZU- FTIR-8400 spectrophotometer using $\mathrm{KBr}$ pellet method. ${ }^{1} \mathrm{H}$ NMR spectra were recorded on Bruker $400 \mathrm{MHz}$ NMR spectrometer in DMSO- $d 6$ with TMS as internal standard. Mass spectrum was recorded on JOEL SX 102/DA-600-Mass spectrometer and elemental analysis was carried out using Heraus $\mathrm{C}, \mathrm{H}$, and $\mathrm{N}$ rapid analyzer

\section{1. General Procedure for synthesis of 5-cyano-1,2,3,4-tetrahydropyrimidine derivatives (RGV-231 to RGV-250)}

A mixture of substituted pyrazole-4-carbaldehyde ( 0.03 mole), ethyl cyanoacetate $(0.03$ mole, $3.39 \mathrm{~g})$, and urea or thiourea $(0.03 \mathrm{~mole})$ in ethanol $(25 \mathrm{~mL})$ containing potassium carbonate $(0.03$ mole, $4.15 \mathrm{~g})$ were refluxed for $4-5 \mathrm{~h}$. The completion of reaction was monitored by TLC. The potassium salt of compound, which precipitated during the reaction, was poured into water and acidified with dilute acetic acid. The precipitates were filtered out, washed thoroughly with water, dried and recrystallized from ethanol to afford the desired compounds

\section{1,2,3,4-tetrahydro-6-(3-(4-nitrophenyl)-1-phenyl-1H-pyrazol-4-yl)-4-oxo-2- thioxopyrimidine-5-carbonitrile (RGV-231)}

M.P. $170-172{ }^{\circ} \mathrm{C}$; IR $\left(\mathrm{cm}^{-1}\right): 3473(\mathrm{~N}-\mathrm{H}$ stretching), $3117(\mathrm{C}-\mathrm{H}$ aromatic), $2196(\mathrm{C} \equiv \mathrm{N}), 1660$ $(\mathrm{C}=\mathrm{O}), 1598(\mathrm{C}=\mathrm{C}), 1580\left(\mathrm{C}-\mathrm{NO}_{2}\right), 1494(\mathrm{Sec} . \mathrm{N}-\mathrm{H}$ def$), 1330(\mathrm{C}-\mathrm{N}), 1230(\mathrm{C}=\mathrm{S}) ;{ }^{1} \mathrm{H}$ NMR (DMSO-d6, $400 \mathrm{MHz}, \delta \mathrm{ppm})$ : 7.32-7.35 (t, 1H, Ar-H), 7.48-7.52 (t, 2H, Ar-H), 7.73 (s, 1H, $\mathrm{H}$ of pyrazole ring), 7.90-7.92 (d, 2H, Ar-H), 7.96 (s, 1H, NH,), $8.11(\mathrm{~s}, 1 \mathrm{H}, \mathrm{NH}), 8.23-8.25$ (d, $2 \mathrm{H}, \mathrm{Ar}-\mathrm{H}), 8.39-8.41$ (d, $2 \mathrm{H}, \mathrm{Ar}-\mathrm{H})$; MS: $m / z$ 416; Anal. Calcd. for $\mathrm{C}_{20} \mathrm{H}_{12} \mathrm{~N}_{6} \mathrm{O}_{3} \mathrm{~S}: \mathrm{C}$, 57.69; H, 2.90; N, 20.18. Found: C, 57.71; H, 3.04; N, 20.25\%.

\section{1,2,3,4-tetrahydro-6-(3-(3-nitrophenyl)-1-phenyl-1H-pyrazol-4-yl)-4-oxo-2- thioxopyrimidine-5-carbonitrile (RGV-232)}

M.P. $184-186{ }^{\circ} \mathrm{C}$; IR $\left(\mathrm{cm}^{-1}\right)$ : $3480(\mathrm{~N}-\mathrm{H}$ stretching), $3110(\mathrm{C}-\mathrm{H}$ aromatic $), 2185(\mathrm{C} \equiv \mathrm{N}), 1672$ $(\mathrm{C}=\mathrm{O}), 1589(\mathrm{C}=\mathrm{C}), 1575\left(\mathrm{C}-\mathrm{NO}_{2}\right), 1512(\mathrm{Sec} . \mathrm{N}-\mathrm{H}$ def $), 1355(\mathrm{C}-\mathrm{N}), 1240(\mathrm{C}=\mathrm{S}) ;{ }^{1} \mathrm{H}$ NMR (DMSO-d6, $400 \mathrm{MHz}, \delta \mathrm{ppm}):$ 7.28-7.31 (t, 1H, Ar-H), 7.51-7.54 (t, 2H, Ar-H), 7.85 (s, 1H, $\mathrm{H}$ of pyrazole ring), 7.87-7.89 (d, 2H, Ar-H), 8.06 (s, 1H, NH,, 8.14 (s, 1H, NH), 8.23-8.27 (m, 3H, Ar-H), 8.51 (s, 1H, Ar-H); MS: m/z 416; Anal. Calcd. for $\mathrm{C}_{20} \mathrm{H}_{12} \mathrm{~N}_{6} \mathrm{O}_{3} \mathrm{~S}: \mathrm{C}, 57.69$; $\mathrm{H}$, $2.90 ; \mathrm{N}, 20.18$. Found: C, 57.73; H, 3.02; N, 20.23\%.

\section{6-(3-(4-chlorophenyl)-1-phenyl-1H-pyrazol-4-yl)-1,2,3,4-tetrahydro-4-oxo-2- thioxopyrimidine-5-carbonitrile (RGV-233)}

M.P. $168-170{ }^{\circ} \mathrm{C}$; IR $\left(\mathrm{cm}^{-1}\right): 3415(\mathrm{~N}-\mathrm{H}$ stretching), $3118(\mathrm{C}-\mathrm{H}$ aromatic $), 2156(\mathrm{C} \equiv \mathrm{N}), 1726$ $(\mathrm{C}=\mathrm{O}), 1631(\mathrm{C}=\mathrm{C}), 1550$ (Sec. N-H def), $1342(\mathrm{C}-\mathrm{N}), 1234(\mathrm{C}=\mathrm{S}), 754(\mathrm{C}-\mathrm{Cl}) ;{ }^{1} \mathrm{H}$ NMR (DMSO-d6, $400 \mathrm{MHz}, \delta \mathrm{ppm}):$ 7.25-7.28 (t, 1H, Ar-H), 7.34-7.38 (t, 2H, Ar-H), 7.65 (s, 1H, $\mathrm{H}$ of pyrazole ring), 7.86-7.88 (d, 2H, Ar-H), 7.92 (s, 1H, NH,, 8.08 (s, 1H, NH), 8.16-8.18 (d, $2 \mathrm{H}, \mathrm{Ar}-\mathrm{H}$ ), 8.33-8.35 (d, $2 \mathrm{H}, \mathrm{Ar}-\mathrm{H})$; MS: $\mathrm{m} / z$ 405; Anal. Calcd. for $\mathrm{C}_{20} \mathrm{H}_{12} \mathrm{ClN}_{5} \mathrm{OS}$ : C, 59.19; H, 2.98; N, 17.26. Found: C, 59.27; H, 2.90; N, 17.12\%. 


\section{1,2,3,4-tetrahydro-6-(3-(4-methoxyphenyl)-1-phenyl-1H-pyrazol-4-yl)-4-oxo-2- thioxopyrimidine-5-carbonitrile (RGV-234)}

M.P. $154-156{ }^{\circ} \mathrm{C}$; IR $\left(\mathrm{cm}^{-1}\right)$ : $3496(\mathrm{~N}-\mathrm{H}$ stretching), $3030(\mathrm{C}-\mathrm{H}$ aromatic $), 2960$ (C-H Alkane), $2216(\mathrm{C} \equiv \mathrm{N}), 1732(\mathrm{C}=\mathrm{O}), 1591(\mathrm{C}=\mathrm{C}), 1508$ (Sec. N-H def), $1296(\mathrm{C}-\mathrm{N}), 1263(\mathrm{C}-$ O-C Aralkyl), $1240(\mathrm{C}=\mathrm{S}) ;{ }^{1} \mathrm{H}$ NMR (DMSO-d6, $\left.400 \mathrm{MHz}, \delta \mathrm{ppm}\right): 3.54$ (s, 3H, $-\mathrm{OCH}_{3}$ ), 7.20-7.23 (t, 1H, Ar-H), 7.30-7.34 (t, 2H, Ar-H), $7.70(\mathrm{~s}, 1 \mathrm{H}, \mathrm{H}$ of pyrazole ring), 7.76-7.78 (d, 2H, Ar-H), 8.02 (s, 1H, NH,), 8.12 (s, 1H, NH), 8.20-8.22 (d, 2H, Ar-H), 8.26-8.29 (d, 2H, Ar-H); MS: $m / z$ 401; Anal. Calcd. for $\mathrm{C}_{21} \mathrm{H}_{15} \mathrm{~N}_{5} \mathrm{O}_{2} \mathrm{~S}$ : C, 62.83; H, 3.77; N, 17.45. Found: $\mathrm{C}$, $62.91 ; \mathrm{H}, 3.83 ; \mathrm{N}, 17.38 \%$.

\section{1,2,3,4-tetrahydro-4-oxo-6-(1-phenyl-3-p-tolyl-1H-pyrazol-4-yl)-2-thioxopyrimidine-5- carbonitrile (RGV-235)}

M.P. $174-176{ }^{\circ} \mathrm{C}$; IR $\left(\mathrm{cm}^{-1}\right)$ : $3478(\mathrm{~N}-\mathrm{H}$ stretching), 3080 (C-H aromatic), 2985 (C-H Alkane), $2208(\mathrm{C} \equiv \mathrm{N}), 1722(\mathrm{C}=\mathrm{O}), 1625(\mathrm{C}=\mathrm{C}), 1514$ (Sec. N-H def), $1315(\mathrm{C}-\mathrm{N}), 1240$ $(\mathrm{C}=\mathrm{S}) ;{ }^{1} \mathrm{H}$ NMR (DMSO-d6, $\left.400 \mathrm{MHz}, \delta \mathrm{ppm}\right): 2.20\left(\mathrm{~s}, 3 \mathrm{H}, \mathrm{CH}_{3}\right), 7.14-7.17(\mathrm{t}, 1 \mathrm{H}, \mathrm{Ar}-\mathrm{H})$, 7.23-7.27 (t, 2H, Ar-H), 7.32-7.34 (d, 2H, Ar-H), 7.42-7.45 (d, 2H, Ar-H), 7.70 (s, 1H, H of pyrazole ring), 7.82-7.84 (d, 2H, Ar-H), $8.02(\mathrm{~s}, 1 \mathrm{H}, \mathrm{NH}),, 8.12(\mathrm{~s}, 1 \mathrm{H}, \mathrm{NH}) ; \mathrm{MS}: m / z 385$; Anal. Calcd. for $\mathrm{C}_{21} \mathrm{H}_{15} \mathrm{~N}_{5} \mathrm{OS}$ : C, 65.44; H, 3.92; N, 18.17. Found: C, 65.58; H, 4.04; N, $18.16 \%$.

\section{6-(3-(4-fluorophenyl)-1-phenyl-1H-pyrazol-4-yl)-1,2,3,4-tetrahydro-4-oxo-2- thioxopyrimidine-5-carbonitrile (RGV-236)}

M.P. 182-184 ${ }^{\circ} \mathrm{C}$; IR $\left(\mathrm{cm}^{-1}\right)$ : $3439(\mathrm{~N}-\mathrm{H}$ stretching), $3055(\mathrm{C}-\mathrm{H}$ aromatic), $2231(\mathrm{C} \equiv \mathrm{N}), 1728$ $(\mathrm{C}=\mathrm{O}), 1620(\mathrm{C}=\mathrm{C}), 1465$ (Sec. N-H def), $1346(\mathrm{C}-\mathrm{N}), 1250(\mathrm{C}=\mathrm{S}), 829(\mathrm{C}-\mathrm{F}) ;{ }^{1} \mathrm{H}$ NMR (DMSO-d6, $400 \mathrm{MHz}, \delta \mathrm{ppm})$ : 7.19-7.22 (t, 1H, Ar-H), 7.30-7.34 (t, 2H, Ar-H), $7.71(\mathrm{~s}, 1 \mathrm{H}$, $\mathrm{H}$ of pyrazole ring), 7.80-7.82 (d, 2H, Ar-H), $7.96(\mathrm{~s}, 1 \mathrm{H}, \mathrm{NH}),, 8.10(\mathrm{~s}, 1 \mathrm{H}, \mathrm{NH}), 8.20-8.22$ (d, $2 \mathrm{H}, \mathrm{Ar}-\mathrm{H}), 8.27-8.29$ (d, $2 \mathrm{H}, \mathrm{Ar}-\mathrm{H})$; MS: $m / z$ 389; Anal. Calcd. for $\mathrm{C}_{20} \mathrm{H}_{12} \mathrm{FN}_{5} \mathrm{OS}$ : C, 61.69; H, 3.11; N, 17.98. Found: C, 61.73; H, 3.21; N, 18.06\%.

\section{6-(3-(4-bromophenyl)-1-phenyl-1H-pyrazol-4-yl)-1,2,3,4-tetrahydro-4-oxo-2- thioxopyrimidine-5-carbonitrile (RGV-237)}

M.P. $188-190^{\circ} \mathrm{C}$; IR $\left(\mathrm{cm}^{-1}\right): 3425(\mathrm{~N}-\mathrm{H}$ stretching), $3066(\mathrm{C}-\mathrm{H}$ aromatic), $2212(\mathrm{C} \equiv \mathrm{N}), 1712$ $(\mathrm{C}=\mathrm{O}), 1625(\mathrm{C}=\mathrm{C}), 1473(\mathrm{Sec} . \mathrm{N}-\mathrm{H}$ def$), 1271(\mathrm{C}-\mathrm{N}), 1250(\mathrm{C}=\mathrm{S}), 586(\mathrm{C}-\mathrm{Br}) ;{ }^{1} \mathrm{H}$ NMR (DMSO-d6, $400 \mathrm{MHz}, \delta \mathrm{ppm}):$ 7.10-7.12 (t, 1H, Ar-H), 7.22-7.24 (t, 2H, Ar-H), 7.65 (s, 1H, $\mathrm{H}$ of pyrazole ring), 7.75-7.77 (d, 2H, Ar-H), 8.05 (s, 1H, NH,, 8.14 (s, 1H, NH), 8.28-8.30 (d, $2 \mathrm{H}, \mathrm{Ar}-\mathrm{H}$ ), 8.41-8.43 (d, $2 \mathrm{H}, \mathrm{Ar}-\mathrm{H})$; MS: $\mathrm{m} / z$ 450; Anal. Calcd. for $\mathrm{C}_{20} \mathrm{H}_{12} \mathrm{BrN}_{5} \mathrm{OS}$ : C, 53.34; H, 2.69; N, 15.55. Found: C, 53.40; H, 2.75; N, 15.63\%.

\section{1,2,3,4-tetrahydro-4-oxo-6-(1,3-diphenyl-1H-pyrazol-4-yl)-2-thioxopyrimidine-5-} carbonitrile (RGV-238)

M.P. $156-158^{\circ} \mathrm{C}$; IR $\left(\mathrm{cm}^{-1}\right): 3450(\mathrm{~N}-\mathrm{H}$ stretching), 3088 (C-H aromatic), $2225(\mathrm{C} \equiv \mathrm{N}), 1693$ $(\mathrm{C}=\mathrm{O}), 1600(\mathrm{C}=\mathrm{C}), 1490$ (Sec. N-H def), $1296(\mathrm{C}-\mathrm{N}), 1272(\mathrm{C}=\mathrm{S}) ;{ }^{1} \mathrm{H}$ NMR (DMSO-d6, $400 \mathrm{MHz}, \delta \mathrm{ppm})$ : 7.05-7.07 (t, 1H, Ar-H), 7.21-7.30 (m, 5H, Ar-H), 7.42-7.44 (t, 2H, Ar-H), $7.74(\mathrm{~s}, 1 \mathrm{H}, \mathrm{H}$ of pyrazole ring), 7.80-7.82 (d, 2H, Ar-H), $8.10(\mathrm{~s}, 1 \mathrm{H}, \mathrm{NH}),, 8.21(\mathrm{~s}, 1 \mathrm{H}, \mathrm{NH})$; MS: $m / z$ 371; Anal. Calcd. for $\mathrm{C}_{20} \mathrm{H}_{13} \mathrm{~N}_{5} \mathrm{OS}$ : C, 64.68; H, 3.53; N, 18.86. Found: C, 66.75; H, 3.60; N, $18.80 \%$. 


\section{1,2,3,4-tetrahydro-6-(3-(2-methoxyphenyl)-1-phenyl-1H-pyrazol-4-yl)-4-oxo-2- thioxopyrimidine-5-carbonitrile (RGV-239)}

M.P. $148-150{ }^{\circ} \mathrm{C}$; IR $\left(\mathrm{cm}^{-1}\right)$ : 3433 (N-H stretching), 3064 (C-H aromatic), 2970 (C-H Alkane), $2235(\mathrm{C} \equiv \mathrm{N}), 1720(\mathrm{C}=\mathrm{O}), 1595(\mathrm{C}=\mathrm{C}), 1490$ (Sec. N-H def), $1280(\mathrm{C}-\mathrm{N}), 1250(\mathrm{C}-$ O-C Aralkyl), $1240(\mathrm{C}=\mathrm{S})$; ${ }^{1} \mathrm{H}$ NMR (DMSO-d6, $\left.400 \mathrm{MHz}, \delta \mathrm{ppm}\right): 3.54\left(\mathrm{~s}, 3 \mathrm{H},-\mathrm{OCH}_{3}\right.$ ), 7.20-7.23 (t, 1H, Ar-H), 7.30-7.34 (t, 2H, Ar-H), 7.70 (s, 1H, H of pyrazole ring), 7.76-7.78 (d, 2H, Ar-H), $8.02(\mathrm{~s}, 1 \mathrm{H}, \mathrm{NH}),, 8.12(\mathrm{~s}, 1 \mathrm{H}, \mathrm{NH}), 8.22(\mathrm{~s}, 1 \mathrm{H}, \mathrm{Ar}-\mathrm{H}), 8.31-8.36(\mathrm{~m}, 3 \mathrm{H}, \mathrm{Ar}-$ H); MS: $m / z$ 401; Anal. Calcd. for $\mathrm{C}_{21} \mathrm{H}_{15} \mathrm{~N}_{5} \mathrm{O}_{2} \mathrm{~S}: \mathrm{C}, 62.83$; $\mathrm{H}, 3.77 ; \mathrm{N}, 17.45$. Found: $\mathrm{C}$, $62.89 ; \mathrm{H}, 3.72 ; \mathrm{N}, 17.40 \%$.

\section{6-(3-(2,4-dichlorophenyl)-1-phenyl-1H-pyrazol-4-yl)-1,2,3,4-tetrahydro-4-oxo-2-} thioxopyrimidine-5-carbonitrile (RGV-240)

M.P. $198-200{ }^{\circ} \mathrm{C}$; IR $\left(\mathrm{cm}^{-1}\right)$ : $3427(\mathrm{~N}-\mathrm{H}$ stretching), 3055 (C-H aromatic), $2220(\mathrm{C} \equiv \mathrm{N}), 1726$ $(\mathrm{C}=\mathrm{O}), 1608(\mathrm{C}=\mathrm{C}), 1479$ (Sec. N-H def), $1292(\mathrm{C}-\mathrm{N}), 1258(\mathrm{C}=\mathrm{S}), 759(\mathrm{C}-\mathrm{Cl}) ;{ }^{1} \mathrm{H}$ NMR (DMSO-d6, $400 \mathrm{MHz}, \delta \mathrm{ppm})$ : 7.14-7.17 (t, 1H, Ar-H), 7.22-7.25 (t, 2H, Ar-H), 7.65 (s, 1H, $\mathrm{H}$ of pyrazole ring), 7.73-7.75 (d, 2H, Ar-H), 8.08 (s, 1H, NH,), 8.14 (s, 1H, NH), 8.24-8.26 (d, $2 \mathrm{H}, \mathrm{Ar}-\mathrm{H}), 8.35$ (s, $1 \mathrm{H}, \mathrm{Ar}-\mathrm{H})$; MS: $\mathrm{m} / z$ 440; Anal. Calcd. for $\mathrm{C}_{20} \mathrm{H}_{11} \mathrm{Cl}_{2} \mathrm{~N}_{5} \mathrm{OS}$ : C, 54.56; H, 2.52; N, 15.91. Found: C, 54.61; H, 2.57; N, 15.96\%.

\section{1,2,3,4-tetrahydro-6-(3-(4-nitrophenyl)-1-phenyl-1H-pyrazol-4-yl)-2,4-dioxopyrimidine-} 5-carbonitrile (RGV-241)

M.P. $174-176^{\circ} \mathrm{C}$; IR $\left(\mathrm{cm}^{-1}\right): 3473(\mathrm{~N}-\mathrm{H}$ stretching), 3117 (C-H aromatic), $2196(\mathrm{C} \equiv \mathrm{N}), 1660$ $(\mathrm{C}=\mathrm{O}), 1598(\mathrm{C}=\mathrm{C}), 1580\left(\mathrm{C}-\mathrm{NO}_{2}\right), 1494$ (Sec. N-H def), $1330(\mathrm{C}-\mathrm{N}) ;{ }^{1} \mathrm{H}$ NMR (DMSO-d6, $400 \mathrm{MHz}, \delta \mathrm{ppm})$ : 7.32-7.35 (t, 1H, Ar-H), 7.48-7.52 (t, 2H, Ar-H), 7.73 (s, 1H, H of pyrazole ring), 7.90-7.92 (d, 2H, Ar-H), $7.96(\mathrm{~s}, 1 \mathrm{H}, \mathrm{NH}),, 8.11(\mathrm{~s}, 1 \mathrm{H}, \mathrm{NH}), 8.23-8.25(\mathrm{~d}, 2 \mathrm{H}, \mathrm{Ar}-\mathrm{H})$, 8.39-8.41 (d, 2H, Ar-H); MS: $m / z$ 400; Anal. Calcd. for $\mathrm{C}_{20} \mathrm{H}_{12} \mathrm{~N}_{6} \mathrm{O}_{4}: \mathrm{C}, 60.00 ; \mathrm{H}, 3.02$; N, 20.99. Found: C, 60.20; H, 3.14; N, 20.89\%.

\section{1,2,3,4-tetrahydro-6-(3-(3-nitrophenyl)-1-phenyl-1H-pyrazol-4-yl)-2,4-dioxopyrimidine- 5-carbonitrile (RGV-242)}

M.P. $180-182^{\circ} \mathrm{C}$; IR $\left(\mathrm{cm}^{-1}\right): 3480(\mathrm{~N}-\mathrm{H}$ stretching), $3110(\mathrm{C}-\mathrm{H}$ aromatic), $2185(\mathrm{C} \equiv \mathrm{N}), 1672$ $(\mathrm{C}=\mathrm{O}), 1589(\mathrm{C}=\mathrm{C}), 1575\left(\mathrm{C}-\mathrm{NO}_{2}\right), 1512(\mathrm{Sec} . \mathrm{N}-\mathrm{H}$ def$), 1355(\mathrm{C}-\mathrm{N}) ;{ }^{1} \mathrm{H}$ NMR (DMSO-d6, $400 \mathrm{MHz}, \delta \mathrm{ppm})$ : 7.28-7.31 (t, 1H, Ar-H), 7.51-7.54 (t, 2H, Ar-H), 7.85 (s, 1H, H of pyrazole ring), 7.87-7.89 (d, 2H, Ar-H), $8.06(\mathrm{~s}, 1 \mathrm{H}, \mathrm{NH}),, 8.14(\mathrm{~s}, 1 \mathrm{H}, \mathrm{NH}), 8.23-8.27(\mathrm{~m}, 3 \mathrm{H}, \mathrm{Ar}-\mathrm{H})$, 8.51 (s, $1 \mathrm{H}, \mathrm{Ar}-\mathrm{H})$; MS: $m / z$ 400; Anal. Calcd. for $\mathrm{C}_{20} \mathrm{H}_{12} \mathrm{~N}_{6} \mathrm{O}_{4}: \mathrm{C}, 60.00 ; \mathrm{H}, 3.02 ; \mathrm{N}, 20.99$. Found: C, 60.13; H, 3.10; N, 20.84\%.

6-(3-(4-chlorophenyl)-1-phenyl-1H-pyrazol-4-yl)-1,2,3,4-tetrahydro-2,4dioxopyrimidine-5-carbonitrile (RGV-243)

M.P. $164-166^{\circ} \mathrm{C}$; IR $\left(\mathrm{cm}^{-1}\right): 3415(\mathrm{~N}-\mathrm{H}$ stretching), $3118(\mathrm{C}-\mathrm{H}$ aromatic), $2156(\mathrm{C} \equiv \mathrm{N}), 1726$ $(\mathrm{C}=\mathrm{O}), 1631(\mathrm{C}=\mathrm{C}), 1550(\mathrm{Sec} . \mathrm{N}-\mathrm{H}$ def $), 1342(\mathrm{C}-\mathrm{N}), 754(\mathrm{C}-\mathrm{Cl}) ;{ }^{1} \mathrm{H}$ NMR (DMSO-d6, 400 $\mathrm{MHz}, \delta \mathrm{ppm})$ : 7.25-7.28 (t, 1H, Ar-H), 7.34-7.38 (t, 2H, Ar-H), $7.65(\mathrm{~s}, 1 \mathrm{H}, \mathrm{H}$ of pyrazole ring), 7.86-7.88 (d, 2H, Ar-H), $7.92(\mathrm{~s}, 1 \mathrm{H}, \mathrm{NH}$ ), $8.08(\mathrm{~s}, 1 \mathrm{H}, \mathrm{NH}), 8.16-8.18(\mathrm{~d}, 2 \mathrm{H}, \mathrm{Ar}-\mathrm{H})$, 8.33-8.35 (d, 2H, Ar-H); MS: $m / z$ 489; Anal. Calcd. for $\mathrm{C}_{20} \mathrm{H}_{12} \mathrm{ClN}_{5} \mathrm{O}_{2}$ : C, 61.63; H, 3.10; N, 17.97. Found: C, $61.51 ; \mathrm{H}, 2.95 ; \mathrm{N}, 18.12 \%$. 


\section{1,2,3,4-tetrahydro-6-(3-(4-methoxyphenyl)-1-phenyl-1H-pyrazol-4-yl)-2,4- dioxopyrimidine-5-carbonitrile (RGV-244)}

M.P. $150-152{ }^{\circ} \mathrm{C}$; IR $\left(\mathrm{cm}^{-1}\right)$ : $3496(\mathrm{~N}-\mathrm{H}$ stretching), 3030 (C-H aromatic), 2960 (C-H Alkane), $2216(\mathrm{C} \equiv \mathrm{N}), 1732(\mathrm{C}=\mathrm{O}), 1591(\mathrm{C}=\mathrm{C}), 1508$ (Sec. N-H def), $1296(\mathrm{C}-\mathrm{N}), 1263(\mathrm{C}-$ O-C Aralkyl); ${ }^{1} \mathrm{H}$ NMR (DMSO-d6, $\left.400 \mathrm{MHz}, \delta \mathrm{ppm}\right): 3.54$ (s, 3H, $\left.-\mathrm{OCH}_{3}\right), 7.20-7.23$ (t, $1 \mathrm{H}$, Ar-H), 7.30-7.34 (t, 2H, Ar-H), 7.70 (s, 1H, H of pyrazole ring), 7.76-7.78 (d, 2H, Ar-H), 8.02 (s, 1H, NH,), $8.12(\mathrm{~s}, 1 \mathrm{H}, \mathrm{NH}), 8.20-8.22(\mathrm{~d}, 2 \mathrm{H}, \mathrm{Ar}-\mathrm{H}), 8.26-8.29(\mathrm{~d}, 2 \mathrm{H}, \mathrm{Ar}-\mathrm{H}) ; \mathrm{MS}: \mathrm{m} / z$ 385; Anal. Calcd. for $\mathrm{C}_{21} \mathrm{H}_{15} \mathrm{~N}_{5} \mathrm{O}_{3}: \mathrm{C}, 65.45 ; \mathrm{H}, 3.92 ; \mathrm{N}, 18.17$. Found: $\mathrm{C}, 65.54 ; \mathrm{H}, 3.83 ; \mathrm{N}$, $18.28 \%$.

\section{1,2,3,4-tetrahydro-2,4-dioxo-6-(1-phenyl-3-p-tolyl-1H-pyrazol-4-yl)pyrimidine-5- carbonitrile (RGV-245)}

M.P. $170-172{ }^{\circ} \mathrm{C}$; IR $\left(\mathrm{cm}^{-1}\right)$ : 3478 (N-H stretching), 3080 (C-H aromatic), 2985 (C-H Alkane), $2208(\mathrm{C} \equiv \mathrm{N}), 1722(\mathrm{C}=\mathrm{O}), 1625(\mathrm{C}=\mathrm{C}), 1514($ Sec. N-H def $), 1315(\mathrm{C}-\mathrm{N})$; ${ }^{1} \mathrm{H}$ NMR (DMSO-d6, $400 \mathrm{MHz}, \delta \mathrm{ppm}): 2.20\left(\mathrm{~s}, 3 \mathrm{H}, \mathrm{CH}_{3}\right), 7.14-7.17$ (t, 1H, Ar-H), 7.23-7.27 (t, 2H, Ar-H), 7.32-7.34 (d, 2H, Ar-H), 7.42-7.45 (d, 2H, Ar-H), 7.70 (s, 1H, H of pyrazole ring), 7.82-7.84 (d, 2H, Ar-H), $8.02(\mathrm{~s}, 1 \mathrm{H}, \mathrm{NH}),$,8.12 (s, 1H, NH),; MS: $m / z$ 369; Anal. Calcd. for $\mathrm{C}_{21} \mathrm{H}_{15} \mathrm{~N}_{5} \mathrm{O}_{2}$ : C, C, 68.28; H, 4.09; N, 18.96. Found: C, 68.39; H, 4.04; N, 18.80\%.

\section{6-(3-(4-fluorophenyl)-1-phenyl-1H-pyrazol-4-yl)-1,2,3,4-tetrahydro-2,4- dioxopyrimidine-5-carbonitrile (RGV-246)}

M.P. $156-158^{\circ} \mathrm{C}$; IR $\left(\mathrm{cm}^{-1}\right): 3439$ (N-H stretching), 3055 (C-H aromatic), $2231(\mathrm{C} \equiv \mathrm{N}), 1728$ $(\mathrm{C}=\mathrm{O}), 1620(\mathrm{C}=\mathrm{C}), 1465$ (Sec. N-H def), $1346(\mathrm{C}-\mathrm{N}), 829(\mathrm{C}-\mathrm{F}) ;{ }^{1} \mathrm{H}$ NMR (DMSO-d6, 400 $\mathrm{MHz}, \delta \mathrm{ppm}):$ 7.19-7.22 (t, 1H, Ar-H), 7.30-7.34 (t, 2H, Ar-H), $7.71(\mathrm{~s}, 1 \mathrm{H}, \mathrm{H}$ of pyrazole ring), 7.80-7.82 (d, 2H, Ar-H), $7.96(\mathrm{~s}, 1 \mathrm{H}, \mathrm{NH}),, 8.10(\mathrm{~s}, 1 \mathrm{H}, \mathrm{NH}), 8.20-8.22(\mathrm{~d}, 2 \mathrm{H}, \mathrm{Ar}-\mathrm{H})$, 8.27-8.29 (d, 2H, Ar-H); MS: $m / z$ 373; Anal. Calcd. for $\mathrm{C}_{20} \mathrm{H}_{12} \mathrm{FN}_{5} \mathrm{O}_{2}: \mathrm{C}, 64.34 ; \mathrm{H}, 3.24 ; \mathrm{N}$, 18.76. Found: C, 64.44; H, 3.21; N, 18.66\%.

\section{6-(3-(4-bromophenyl)-1-phenyl-1H-pyrazol-4-yl)-1,2,3,4-tetrahydro-2,4- dioxopyrimidine-5-carbonitrile (RGV-247)}

M.P. $178-180^{\circ} \mathrm{C}$; IR $\left(\mathrm{cm}^{-1}\right)$ : $3425(\mathrm{~N}-\mathrm{H}$ stretching), $3066(\mathrm{C}-\mathrm{H}$ aromatic), $2212(\mathrm{C} \equiv \mathrm{N}), 1712$ $(\mathrm{C}=\mathrm{O}), 1625(\mathrm{C}=\mathrm{C}), 1473$ (Sec. N-H def), $1271(\mathrm{C}-\mathrm{N}), 586(\mathrm{C}-\mathrm{Br}) ;{ }^{1} \mathrm{H}$ NMR (DMSO-d6, 400 $\mathrm{MHz}, \delta \mathrm{ppm})$ : 7.10-7.12 (t, 1H, Ar-H), 7.22-7.24 (t, 2H, Ar-H), 7.65 (s, 1H, H of pyrazole ring), 7.75-7.77 (d, 2H, Ar-H), $8.05(\mathrm{~s}, 1 \mathrm{H}, \mathrm{NH}),, 8.14(\mathrm{~s}, 1 \mathrm{H}, \mathrm{NH}), 8.28-8.30(\mathrm{~d}, 2 \mathrm{H}, \mathrm{Ar}-\mathrm{H})$, 8.41-8.43 (d, $2 \mathrm{H}$, Ar-H); MS: $m / z$ 434; Anal. Calcd. for $\mathrm{C}_{20} \mathrm{H}_{12} \mathrm{BrN}_{5} \mathrm{O}_{2}$ : C, 55.32; H, 2.79; N, 16.13. Found: C, 55.40; H, 2.75; N, 16.27\%.

\section{1,2,3,4-tetrahydro-2,4-dioxo-6-(1,3-diphenyl-1H-pyrazol-4-yl)pyrimidine-5-carbonitrile (RGV-248)}

M.P. $160-162{ }^{\circ} \mathrm{C}$; IR $\left(\mathrm{cm}^{-1}\right): 3450$ (N-H stretching), 3088 (C-H aromatic), $2225(\mathrm{C} \equiv \mathrm{N}), 1693$ $(\mathrm{C}=\mathrm{O}), 1600(\mathrm{C}=\mathrm{C}), 1490$ (Sec. N-H def), $1296(\mathrm{C}-\mathrm{N}) ;{ }^{1} \mathrm{H}$ NMR (DMSO-d6, $400 \mathrm{MHz}$, Sppm): 7.05-7.07 (t, 1H, Ar-H), 7.21-7.30 (m, 5H, Ar-H), 7.42-7.44 (t, 2H, Ar-H), 7.74 (s, $1 \mathrm{H}, \mathrm{H}$ of pyrazole ring), 7.80-7.82 (d, 2H, Ar-H), $8.10(\mathrm{~s}, 1 \mathrm{H}, \mathrm{NH}),, 8.21(\mathrm{~s}, 1 \mathrm{H}, \mathrm{NH})$; MS: $m / z$ 355; Anal. Calcd. for $\mathrm{C}_{20} \mathrm{H}_{13} \mathrm{~N}_{5} \mathrm{O}_{2}$ : C, 67.60; H, 3.69; N, 19.71. Found: C, 67.75; H, 3.75; $\mathrm{N}, 19.80 \%$. 


\section{1,2,3,4-tetrahydro-6-(3-(2-methoxyphenyl)-1-phenyl-1H-pyrazol-4-yl)-2,4- dioxopyrimidine-5-carbonitrile (RGV-249)}

M.P. $142-144{ }^{\circ} \mathrm{C}$; IR $\left(\mathrm{cm}^{-1}\right)$ : 3433 (N-H stretching), 3064 (C-H aromatic), 2970 (C-H Alkane), $2235(\mathrm{C} \equiv \mathrm{N}), 1720(\mathrm{C}=\mathrm{O}), 1595(\mathrm{C}=\mathrm{C}), 1490$ (Sec. N-H def), $1280(\mathrm{C}-\mathrm{N}), 1250(\mathrm{C}-$ O-C Aralkyl); ${ }^{1} \mathrm{H}$ NMR (DMSO-d6, $\left.400 \mathrm{MHz}, \delta \mathrm{ppm}\right): 3.54$ (s, 3H, $\left.-\mathrm{OCH}_{3}\right), 7.20-7.23$ (t, $1 \mathrm{H}$, Ar-H), 7.30-7.34 (t, 2H, Ar-H), 7.70 (s, 1H, H of pyrazole ring), 7.76-7.78 (d, 2H, Ar-H), 8.02 (s, 1H, NH,), $8.12(\mathrm{~s}, 1 \mathrm{H}, \mathrm{NH}), 8.22(\mathrm{~s}, 1 \mathrm{H}, \mathrm{Ar}-\mathrm{H}), 8.31-8.36(\mathrm{~m}, 3 \mathrm{H}, \mathrm{Ar}-\mathrm{H})$; MS: $\mathrm{m} / \mathrm{z} 385$; Anal. Calcd. for $\mathrm{C}_{21} \mathrm{H}_{15} \mathrm{~N}_{5} \mathrm{O}_{3}: \mathrm{C}, 65.45 ; \mathrm{H}, 3.92 ; \mathrm{N}, 18.17$. Found: $\mathrm{C}, 66.62 ; \mathrm{H}, 3.82 ; \mathrm{N}$, $18.30 \%$.

\section{6-(3-(2,4-dichlorophenyl)-1-phenyl-1H-pyrazol-4-yl)-1,2,3,4-tetrahydro-2,4- dioxopyrimidine-5-carbonitrile (RGV-250)}

M.P. $190-192{ }^{\circ} \mathrm{C}$; IR $\left(\mathrm{cm}^{-1}\right)$ : 3427 (N-H stretching), 3055 (C-H aromatic), $2220(\mathrm{C} \equiv \mathrm{N}), 1726$ $(\mathrm{C}=\mathrm{O}), 1608(\mathrm{C}=\mathrm{C}), 1479$ (Sec. N-H def), $1292(\mathrm{C}-\mathrm{N}), 759(\mathrm{C}-\mathrm{Cl}) ;{ }^{1} \mathrm{H}$ NMR (DMSO-d6, 400 $\mathrm{MHz}, \delta \mathrm{ppm})$ : 7.14-7.17 (t, 1H, Ar-H), 7.22-7.25 (t, 2H, Ar-H), 7.65 (s, 1H, H of pyrazole ring), 7.73-7.75 (d, 2H, Ar-H), $8.08(\mathrm{~s}, 1 \mathrm{H}, \mathrm{NH}$ ), $8.14(\mathrm{~s}, 1 \mathrm{H}, \mathrm{NH}), 8.24-8.26(\mathrm{~d}, 2 \mathrm{H}, \mathrm{Ar}-\mathrm{H})$, 8.35 (s, $1 \mathrm{H}, \mathrm{Ar}-\mathrm{H})$; MS: $\mathrm{m} / z$ 424; Anal. Calcd. for $\mathrm{C}_{20} \mathrm{H}_{11} \mathrm{Cl}_{2} \mathrm{~N}_{5} \mathrm{O}_{2}$ : C, 56.62; $\mathrm{H}, 2.61 ; \mathrm{N}$, 16.51. Found: $\mathrm{C}, 56.70 ; \mathrm{H}, 2.57 ; \mathrm{N}, 16.66 \%$.

\section{CONCLUSION}

In conclusion, we have developed a highly efficient base catalyzed, one pot, three component protocol for the synthesis of 5-cyano-1,2,3,4-tetrahydropyrimidine derivatives via condensation of substituted pyrazole-4-carbaldehyde, ethylcyano acetate and thiourea/urea in the presence of base catalyst. The advantages of this method are clean reaction, short reaction time, high yield, easy purification and economic availability of the catalyst.

\section{Acknowledgement}

Authors are thankful to the Professor and Head, Department of Chemistry, Saurashtra University-Rajkot for providing necessary laboratory facilities.

\section{References}

[1] D. M. D'Souza, T. J. Müller, Chem. Soc. Rev. 36 (2007) 1095.

[2] A. Domling, Chem. Rev. 106 (2006) 17.

[3] I. Ugi, Pure and Appl. Chem. 73 (2001) 187.

[4] Sharma, P.; Rane, N.; Gurram, V. K. Bioorg. Med. Chem. Lett. 14 (2004) 4185.

[5] Ito S., Masuda K., Kusano S., Nagata T., Kojima Y., Sawai N., Maeno S., US 4988704 A, 1991.

[6] Ram V. J., Haque N., Guru P. Y., Eur. J. Med. Chem. 27 (1992) 851.

[7] Amir M., Javed S., Kumar H., Indian J. Pharm. Sci. 69 (2007) 337. 
[8] Vega S., Alonso J., Diaz J. A., Junquera F., J. Heterocyclic Chem. 27 (1990) 269.

[9] Hannah D. R., Stevens M. F. G., J CHEM RES 2003 (2003) 398.

[10] Smith, P. A. S.; Kan, R. O. J. Org. Chem. 29 (1964) 2261.

[11] Balzarini, J.; McGuigan, C. J. Antimicrob. Chemother. 50 (2002) 5.

[12] Lee, H. W.; Kim, B. Y.; Ahn, J. B.; Kang, S. K.; Lee, J. H.; Shin, J. S.; Ahn, S. K.; Lee, S. J.; Yoon, S. S. Eur. J. Med. Chem.40 (2005) 862.

[13] Juby, P. F.; Hudyma, T. W.; Brown, M.; Essery, J. M.; Partyka, R. A. J. Med. Chem. 22 (1979) 263.

[14] Gupta, A.; Kayath, H.; Ajit, S.; Geeta, S.; Mishra, K. Indian J. Pharmacol. 26 (1994) 227.

[15] Abu-Hashem, A. A.; Youssef, M. M.; Hussein, H. A. R. J. Chinese Chem. Soc. 58 (2011) 41.

[16] Rahaman, S. A.; Rajendra Pasad, Y.; Kumar, P.; Kumar, B. Saudi Pharm J 17 (2009) 255.

[17] Nezu, Y.; Miyazaki, M.; Sugiyama, K.; Kajiwara, I. Pestic Sci 47 (1996) 103.

[18] Coe, J. W.; Fliri, A. F. J.; Kaneko, T.; Larson, E. R.; US 5491234 A, 1996.

[19] Rodrigues, A. L. S.; Rosa, J. M.; Gadotti, V. M.; Goulart, E. C.; Santos, M. M.; Silva, A. V.; Sehnem, B.; Rosa, L. S.; Gonçalves, R. M.; Corrêa, R.; Santos, A. R. S. Pharmacol Biochem Be. 82 (2005) 156.

[20] Kumar, B.; Kaur, B.; Kaur, J.; Parmar, A.; Anand, R.; Kumar, H. Indian J Chem B 41 (2002) 1526.

[21] Murthy, Y. L.; Saviri, R. M. R.; Parimi, A. R.; Nareesh, S. Organic Communications 6 (2013).

[22] Abdou, I. M.; Strekowski, L. Tetrahedron 56 (2000) 8631.

[23] Fathalla, O.; Zeid, I.; Haiba, M.; Soliman, A.; Abd-Elmoez, S. I.; El-Serwy, W. World J. Chem 4 (2009) 127.

[24] Pore Y., Kuchekar B., Dig J Nano Biostr 3 (2008) 293. 